\title{
Triple Product Integrals of Laguerre Functions
}

\author{
By J. Gillis and M. Shimshoni
}

1. Introduction. We shall use the following standard definitions for Laguerre polynomials (1) and Laguerre functions (2):

$$
\begin{gathered}
L_{n}(x)=\sum_{r=0}^{n}(-1)^{r}\left(\begin{array}{l}
n \\
r
\end{array}\right) \frac{x^{r}}{r !} \\
\lambda_{n}(x)=e^{-x / 2} L_{n}(x)
\end{gathered}
$$

The Laguerre functions are known to constitute a complete orthonormal set in $L^{2}(0, \infty)$. Given a differential equation over $0 \leqq x<\infty$ one naturally thinks, therefore, of the possibility of solution by expansion as a series of Laguerre functions. However, for this to be useful for non-linear differential equations, we need to be able to expand the product of two Laguerre functions as a linear series of these functions. The main part of this paper is devoted to methods for effecting the expansion, and we shall also give an application of the results. The similar problem for Laguerre polynomials has been solved by Watson [5], and methods for computing the expansion coefficients discussed by Gillis and Weiss [3].

We may write

$$
\lambda_{r} \lambda_{s}=\sum_{t=0}^{\infty} C_{r s t} \lambda_{t}
$$

where

$$
C_{r s t}=\int_{0}^{\infty} \lambda_{r}(x) \lambda_{s}(x) \lambda_{t}(x) d x
$$

We shall also write this as $C_{r, s, t}$.

It follows that the coefficients will be symmetric in all three suffixes. We give below a table of these coefficients for $0 \leqq r \leqq s \leqq t \leqq 10$, and also expressions of $C_{r s t}$ as polynomials in $t$ for $0 \leqq r \leqq s \leqq 3$.

In Section 2 we discuss a number of formulas for $C_{r s t}$. Those in (a) and (b) involve three-fold summations and apply to general $r, s, t$. In (c) we obtain a simple sum formula valid for the case $r=0$ and, in (d), a double sum for the case $r=s$. In Section 3 we shall derive two recurrence formulas for the coefficients $C_{r s t}$. As a check on the stability of these latter formulas in practice, it is advisable to have comparatively simple alternative methods for computing ${ }^{\prime} C_{r s t}$ for certain particular triads $r, s, t$. For this purpose the formulas of Section 2 (c), (d) can be of use.

\section{Explicit Formulas.}

(a)

$$
\begin{aligned}
C_{r s t} & =\int_{0}^{\infty} e^{-3 x / 2} \sum_{\alpha=0}^{r}(-1)^{\alpha}\left(\begin{array}{c}
r \\
\alpha
\end{array}\right) \frac{x^{\alpha}}{\alpha !} \sum_{\beta=0}^{s}(-1)^{\beta}\left(\begin{array}{c}
s \\
\beta
\end{array}\right) \frac{x^{\beta}}{\beta !} \sum_{\gamma=0}^{t}(-1)^{\gamma}\left(\begin{array}{l}
t \\
\gamma
\end{array}\right) \frac{x^{\gamma}}{\gamma !} d x \\
& =\frac{2}{3} \sum_{\alpha, \beta, \gamma}\left(-\frac{2}{3}\right)^{\alpha+\beta+\gamma}\left(\begin{array}{c}
r \\
\alpha
\end{array}\right)\left(\begin{array}{l}
s \\
\beta
\end{array}\right)\left(\begin{array}{l}
t \\
\gamma
\end{array}\right) \frac{(\alpha+\beta+\gamma) !}{\alpha ! \beta ! \gamma !}
\end{aligned}
$$

Received May 8, 1961. This research has been supported by the Air Research and Development Command of the U. S. Air Force (European Office). 
(b) Since

$$
x^{n}=n ! \sum_{\alpha=0}^{n}(-1)^{\alpha}\left(\begin{array}{l}
n \\
\alpha
\end{array}\right) L_{\alpha}(\dot{x}), \quad(\text { cf. }[1])
$$

it follows that

$$
\begin{aligned}
L_{r}(x) L_{s}(x) & =\sum_{p=0}^{r}(-1)^{p}\left(\begin{array}{l}
r \\
p
\end{array}\right) \frac{x^{p}}{p !} \sum_{q=0}^{s}(-1)^{q}\left(\begin{array}{l}
s \\
q
\end{array}\right) \frac{x^{q}}{q !} \\
& =\sum_{p=0}^{r} \sum_{q=0}^{s}(-1)^{p+q}\left(\begin{array}{l}
r \\
p
\end{array}\right)\left(\begin{array}{c}
s \\
q
\end{array}\right)\left(\begin{array}{c}
p+q \\
p
\end{array}\right) \sum_{t=0}^{p+q}(-1)^{t}\left(\begin{array}{c}
p+q \\
t
\end{array}\right) L_{t}(x),
\end{aligned}
$$

i.e.,

$$
L_{r}(x) L_{s}(x)=\sum_{t} A_{r s t} L_{t}(x)
$$

where

$$
A_{r s t}=\sum_{p=0}^{r} \sum_{q=0}^{s}(-1)^{p+q+t}\left(\begin{array}{l}
r \\
p
\end{array}\right)\left(\begin{array}{c}
s \\
q
\end{array}\right)\left(\begin{array}{c}
p+q \\
p
\end{array}\right)\left(\begin{array}{c}
p+q \\
t
\end{array}\right)
$$

Now [1],

$$
e^{-x / 2}=2 \sum_{p=0}^{\infty} 3^{-p-1} L_{p}(x)
$$

and so

$$
\begin{aligned}
\sum_{t} C_{r s t} L_{t}(x)=e^{-x / 2} L_{r}(x) L_{s}(x) & =2 \sum_{p=0}^{\infty} \sum_{m=0}^{\infty} 3^{-p-1} A_{r s m} L_{p}(x) L_{m}(x) \\
& =2 \sum_{p=0}^{\infty} \sum_{m=0}^{\infty} \sum_{t=|m-p|}^{m+p} 3^{-p-1} A_{r s m} A_{m p t} L_{t}(x)
\end{aligned}
$$

Hence

$$
\begin{aligned}
& C_{r s t}=2 \sum_{p, m} 3^{-p-1} A_{r s m} A_{m p t}=2 \sum_{m, p, \alpha, \beta, \gamma, \delta}(-1)^{\alpha+\beta+\gamma+\delta+m+t} 3^{-p-1} \\
& \cdot\left(\begin{array}{l}
r \\
\alpha
\end{array}\right)\left(\begin{array}{c}
s \\
\beta
\end{array}\right)\left(\begin{array}{c}
\alpha+\beta \\
\alpha
\end{array}\right)\left(\begin{array}{c}
\alpha+\beta \\
m
\end{array}\right)\left(\begin{array}{c}
m \\
\gamma
\end{array}\right)\left(\begin{array}{c}
p \\
\delta
\end{array}\right)\left(\begin{array}{c}
\gamma+\delta \\
\gamma
\end{array}\right)\left(\begin{array}{c}
\gamma+\delta \\
t
\end{array}\right)
\end{aligned}
$$

The sum over $p$ yields

$$
\sum_{p \geqq \delta}\left(\begin{array}{l}
p \\
\delta
\end{array}\right) 3^{-p-1}=2^{-\delta-1}
$$

while the sum over $m$ is

$$
\begin{aligned}
& \sum_{m}(-1)^{m}\left(\begin{array}{c}
\alpha+\beta \\
m
\end{array}\right)\left(\begin{array}{c}
m \\
\gamma
\end{array}\right)=(-1)^{\gamma}\left(\begin{array}{c}
\alpha+\beta \\
\gamma
\end{array}\right) \sum_{m}(-1)^{m-\gamma}\left(\begin{array}{c}
\alpha+\beta-\gamma \\
m-\gamma
\end{array}\right) \\
&=0 \text { if } \gamma \neq \alpha+\beta \\
&=(-1)^{\gamma} \text { if } \gamma=\alpha+\beta
\end{aligned}
$$

Hence

$$
C_{r s t}=\sum_{\alpha=0}^{r} \sum_{\beta=0}^{\delta} \sum_{\delta=0}^{\infty} \frac{(-1)^{\alpha+\beta+t+\delta}}{2^{\delta}}\left(\begin{array}{l}
r \\
\alpha
\end{array}\right)\left(\begin{array}{l}
s \\
\beta
\end{array}\right)\left(\begin{array}{c}
\alpha+\beta \\
\alpha
\end{array}\right)\left(\begin{array}{c}
\alpha+\beta+\delta \\
\delta
\end{array}\right)\left(\begin{array}{c}
\alpha+\beta+\delta \\
t
\end{array}\right)
$$


(c) Take Laplace transforms of both sides of (3). This gives [2]

$$
\begin{aligned}
\left(\begin{array}{c}
r+s \\
r
\end{array}\right) p^{r+s}(p-1)^{-r-s-1}{ }_{2} F_{1}[-r,-s ;-r & \left.-s ; 1-p^{-2}\right] \\
& =\sum_{t} C_{r s t}\left(p-\frac{1}{2}\right)^{t}\left(p+\frac{1}{2}\right)^{-t-1}
\end{aligned}
$$

Writing $q=\left(p-\frac{1}{2}\right) /\left(p+\frac{1}{2}\right)$, we obtain

$$
\begin{aligned}
\sum_{t} C_{r s t} q^{t}=2\left(\begin{array}{c}
r+s \\
r
\end{array}\right)(1+q)^{r+s} & (3-q)^{-r-s-1} \\
\cdot{ }_{2} F_{1} & {\left[-r,-s ;-r-s ; \frac{(3-q)(3 q-1)}{(q+1)^{2}}\right] . }
\end{aligned}
$$

In the special case $r=0$ this leads to the simple result that

$$
\begin{aligned}
C_{0 s t}=\text { coefficient of } q^{t} \text { in } 2(1+q)^{s}(3-q)^{-s-1} \\
=2 \sum_{n=0}^{t} 3^{-n-s-1}(s+n) !(n !(t-n) !(s+n-t) !\}^{-1}
\end{aligned}
$$

We could similarly use (16) to obtain expressions for $C_{r s t}$ for small nonzero values of $r$, but the formulas soon become prohibitively complicated. It follows incidentally from (17) that $C_{0 s t}>0$ for all $s, t$ and that $3^{s+t+1} C_{0 s i}$ is an even integer.

(d) From the generating function [1]

$$
\sum_{n=0}^{\infty} L_{n}(z) u^{n}=(1-u)^{-1} \exp \left\{u z(u-1)^{-1}\right\}
$$

we obtain

$$
\sum_{n=0}^{\infty} \lambda_{n}(z) u^{n}=(1-u)^{-1} \exp \left\{\frac{1}{2} z(u+1)(u-1)^{-1}\right\}
$$

Hence

$$
\begin{aligned}
\lambda_{r}(z) \lambda_{s}(z)=(2 \pi i)^{-2} \int^{\left(0^{+}\right)} \int^{\left(0^{+}\right)}(1-u)^{-1}(1-v)^{-3} u^{-r-1} v^{-s-1} \\
\cdot \exp \left\{\frac{1}{2} z\left[\frac{u+1}{u-1}+\frac{v+1}{v-1}\right]\right\} d u d v .
\end{aligned}
$$

Choose $w$ so that

$$
(w+1) /(w-1)=(u+1) /(u-1)+(v+1) /(v-1)
$$

and write

$$
\exp \left\{\frac{1}{2}(w+1)(w-1)^{-1}\right\}=(1-w) \sum_{t=0}^{\infty} \lambda_{t}(z) w^{t}
$$

Then

$$
\lambda_{r}(z) \lambda_{s}(z)=\sum_{t=0}^{\infty}(2 \pi i)^{-2} \lambda_{t}(z) \int^{\left(0^{+}\right)} \int^{0(+)} F_{t}(u, v) u^{-r-1} v^{-s-1} d u d v
$$


where

$$
F_{t}(u, v)=2(1+u+v-3 u v)^{t}(3-u-v-u v)^{-t-1} .
$$

It follows that $C_{r s t}$ is the coefficient of $u^{r} v^{s}$ in $F_{t}(u, v)$.

By putting $u=0$ we can calculate $C_{0 s t}$ and immediately recover (17). Formulas for other small values of $r$ can be obtained by expanding $\left[\frac{\partial^{r}}{\partial u^{r}} F_{t}(u, v)\right]_{u=0}$ in powers of $v$. However, there is another case in which this can be used to obtain a closed expression for $C_{r s t}$, namely, when $r=s$. For this purpose we write

$$
F_{t}(u, v)=(-1)^{t} 2^{-2 t-1}(4 \alpha-\beta)^{t}\left(1-\frac{1}{4} \beta\right)^{-t-1}
$$

where

$$
a=u v
$$

and

$$
\beta=(u+1)(v+1)
$$

We note that the coefficient of $u^{r} v^{r}$ in the expansion of $\beta^{n}$ is $\left(\begin{array}{l}n \\ r\end{array}\right)^{2}$. But, by (24),

$$
F_{t}(u, v)=(-1)^{t} 2^{-2 t-1} \sum_{m=0}^{t} \sum_{n=0}^{\infty}(-1)^{m}\left(\begin{array}{c}
t \\
m
\end{array}\right)(4 \alpha)^{t-m} \beta^{m+n} 4^{-n}\left(\begin{array}{c}
t+n \\
n
\end{array}\right) .
$$

It follows that $C_{r r t}$, the coefficient of $u^{r} v^{r}$ in the expression $F_{t}(u, v)$, is

$$
\frac{1}{2}(-1)^{t} \sum_{m=0}^{t} \sum_{n=0}^{\infty}(-1)^{m} 2^{-2(m+n)}\left(\begin{array}{c}
t \\
m
\end{array}\right)\left(\begin{array}{c}
t+n \\
n
\end{array}\right)\left(\begin{array}{c}
m+n \\
r-t+m
\end{array}\right)^{2} .
$$

The table of numerical values of $C_{r s t}$ suggests the conjecture that $C_{r r t}$ has the sign of $(-1)^{t}$ for $0 \leqq t \leqq r$. This would mean that the double sum in (27) is always positive for $0 \leqq t \leqq r$, but we have not been able to prove the conjecture.

\section{Recurrence Relations.}

(a) A Two-Index Recurrence Relation. From the relation

$$
x \lambda_{r}(x)=-(r+1) \lambda_{r+1}(x)+(2 r+1) \lambda_{r}(x)-r \lambda_{r-1}(x),
$$

[1], we deduce that

$$
\int_{0}^{\infty} x \lambda_{r}(x) \lambda_{r+1}(x) d x=-(r+1)
$$

and

$$
\int_{0}^{\infty} x \lambda_{r}^{2} d x=2 r+1
$$

while

$$
\int_{0}^{\infty} x \lambda_{r} \lambda_{s} d x=0 \quad \text { if } s \neq r, r \pm 1 .
$$


Multiplying both sides of (28) by $\lambda_{s}$ and using (3), we get

$$
\begin{aligned}
\sum_{t=0}^{\infty} C_{r s t} x \lambda_{t} & \\
= & -(r+1) \sum_{t=0}^{\infty} C_{r+1, s, t} \lambda_{t}+(2 r+1) \sum_{t=0}^{\infty} C_{r s t} \lambda_{t}-r \sum_{t=0}^{\infty} C_{r-1, s, t} \lambda_{t} .
\end{aligned}
$$

We now multiply both sides of (32) by $\lambda_{t}$ and integrate from 0 to $\infty$, using (29), (30), (31), and immediately obtain

$$
\begin{aligned}
& (r+1) C_{r+1,8, t} \\
& \quad=t C_{r, s, t-1}+2(r-t) C_{r, 8, t}+(t+1) C_{r, 8, t+1}-r C_{r-1,8, t} .
\end{aligned}
$$

This is the required recurrence relation. It can be used quite effectively, in conjunction with (17), to compute a table of $C_{r s t}$. We first compute $C_{0 s t}$ by (17) for an adequate range of $(s, t)$. It then follows from (33) that

$$
C_{1 s t}=t C_{0,8, t-1}-2 t C_{0 s t}+(t+1) C_{0,8, t+1}
$$

and this gives us $C_{1 s t}$, etc. The computation is reasonably stable, although, as a safety precaution, one would carry more digits than are actually needed. Actually, the explicit formula for $C_{1 s t}$, obtainable from (17) and (34), is

$$
C_{1 s t}=2 \sum_{n=0}^{t} 3^{-n-s-2}(s-1+n) !\{n !(t-n) !(s-t+n+1) !\}^{-1} F_{n s t}
$$

where

$$
\begin{aligned}
F_{n s t}=(4 s+1) n^{2}+\left(8 s^{2}-12 s t\right. & +6 s+1) n \\
& +s\left[4 s^{2}-(12 t-5) s+9 t(t-1)+1\right] .
\end{aligned}
$$

A similar, but much more complicated formula can be derived from this for $C_{2 s t}$. The corresponding formulas for $C_{r s t}$ become very complicated for larger values of $r$.

For work with an electronic computer it would be better to have a method for generating the $C_{r s t}$ as required rather than to store a table of these coefficients. In the next paragraph we propose a method of achieving this by a recurrence relation which operates on only one of the indices.

(b) An Alternative Recurrence Relation. We recall that $\lambda_{r}$ satisfies the differential equation

$$
x \lambda_{r}{ }^{\prime \prime}+\lambda_{r}{ }^{\prime}+\left(r+\frac{1}{2}-\frac{1}{4} x\right) \lambda_{r}=0 .
$$

Hence, if we write

$$
u_{r}=x^{1 / 2} \lambda_{r}
$$

we can easily verify that

$$
u_{r}^{\prime \prime}+P_{r} u_{r}=0
$$


where

$$
P_{r}=\frac{1}{4 x^{2}}+\frac{r+\frac{1}{2}}{x}-\frac{1}{4}
$$

It may then be deduced, by a standard procedure, that $y=u_{r} u_{s}$ satisfies the differential equation

$$
\begin{aligned}
x^{4} y^{(I V)}+x^{3} y^{\prime \prime}+x^{2}\left(1+2 \sigma x-x^{2}\right) y^{\prime \prime} & \\
& -x\left(2+\sigma x+x^{2}\right) y^{\prime}+\left(2+\sigma x+\delta^{2} x^{2}\right) y=0
\end{aligned}
$$

where

$$
\left.\begin{array}{l}
\sigma=r+s+1 \\
\delta=|r-s|
\end{array}\right\} .
$$

Let $z=\lambda_{r} \lambda_{s}$. Then, by (38),

$$
y=x z
$$

and this may be substituted directly into (41), giving

(44) $\mathscr{L}(z)=x^{2} z^{(1 V)}+5 x z^{m}+\left(4+2 \sigma x-x^{2}\right) z^{\prime \prime}+3(\sigma-x) z^{\prime}+\left(\delta^{2}-1\right) z=0$.

We now write

$$
z=\sum_{t} C_{r s t} \lambda_{t}
$$

and substitute in $\mathscr{L}(z)$.

After a little manipulation, using the properties of Laguerre functions, we obtain (46) $\mathscr{L}\left(\lambda_{t}\right)=\left(\sigma-\rho-\frac{5 x}{4}\right) \lambda_{t}{ }^{\prime}+\left[\rho^{2}-2 \rho \sigma+\delta^{2}-\frac{1}{2}+\frac{\sigma+\rho}{2} x-\frac{3}{16} x^{2}\right] \lambda_{t}$ where

$$
\rho=t+\frac{1}{2}
$$

and hence, again going back to basic properties of $\lambda_{t}$,

$$
\begin{aligned}
x \mathscr{L}\left(\lambda_{t}\right)=\frac{3}{16}( & +1)(t+2)(t+3) \lambda_{t+3} \\
& -\frac{1}{16}(t+1)(t+2)(10 t-8 \sigma+13) \lambda_{t+2} \\
& -\frac{1}{16}(t+1)\left[3 t^{2}+2 t+2\left(8 \delta^{2}+4 \sigma+1\right)\right] \lambda_{t+1} \\
& +\left[\frac{5}{8}(2 t+1)\left(t^{2}+t+1\right)+\delta^{2}(t+1)-\sigma\left(t^{2}+t+\frac{1}{2}\right)\right] \lambda_{t} \\
& -\frac{1}{16} t\left(3 t^{2}+4 t+16 \delta^{2}-8 \sigma+3\right) \lambda_{t-1} \\
& -\frac{1}{16} t(t-1)(10 t-8 \sigma-3) \lambda_{t-2}+\frac{3}{16} t(t-1)(t-2) \lambda_{t-3} .
\end{aligned}
$$

It follows from (45) and (47) that

$$
x \mathscr{L}(z)=\sum B_{t} \lambda_{t} .
$$


where

$$
\begin{aligned}
B_{t}=\frac{3}{16}(t+ & 3)(t+2)(t+1) C_{r, s, t+3} \\
& -\frac{1}{16}(t+2)(t+1)(10 t-8 \sigma+17) C_{r, s, t+2} \\
& -\frac{1}{16}(t+1)\left[3 t^{2}+10 t+16 \delta^{2}-8 \sigma+10\right] C_{r, s, t+1} \\
& +\left[\frac{5}{8}(2 t+1)\left(t^{2}+t+1\right)+\delta^{2}(2 t+1)-\sigma\left(t^{2}+t+\frac{1}{2}\right)\right] C_{r s t} \\
& -\frac{1}{16} t\left[3 t^{2}+4 t+16 \delta^{2}+8 \sigma+3\right] C_{r, s, t-1} \\
& -\frac{1}{16} t(t-1)(10 t-8 \sigma-7) C_{r, s, t-2} \\
& +\frac{3}{16} t(t-1)(t-2) C_{r, 8, t-3} .
\end{aligned}
$$

Since $\mathcal{L}(z)=0$ we have, from the completeness of the $\lambda_{i}$ 's, that

$$
\begin{aligned}
C_{r, s, t+3} & =\frac{10 t-8 \sigma+17}{3(t+3)} C_{r, s, t+2}+\frac{3 t^{2}+10 t+16 \delta^{2}-8 \sigma+10}{3(t+2)(t+3)} C_{r, s, t+1} \\
& -\frac{10\left(2 t^{3}+3 t^{2}+3 t+1\right)+2 \delta^{2}(2 t+1)-16 \sigma\left(t^{2}+t+\frac{1}{2}\right)}{3(t+1)(t+2)(t+3)} C_{r, 8, t} \\
& +\frac{t\left(3 t^{2}-4 t+16 \delta^{2}+8 \sigma+3\right)}{3(t+1)(t+2)(t+3)} C_{r, s, t-1} \\
& +\frac{t(t-1)(10 t-8 \sigma-7)}{3(t+1)(t+2)(t+3)} C_{r, 8, t-2}-\frac{t(t-1)(t-2)}{(t+1)(t+2)(t+3)} C_{r, 8, t-3} .
\end{aligned}
$$

Suppose, then, we know $C_{r s t}$ for $t=0,1,2$. Then, putting $t=0$ in (50) gives us

$$
\begin{aligned}
18 C_{r, 8,3}=-2(8 \sigma-17) & C_{r, 8,2} \\
& +2\left(8 \delta^{2}-4 \sigma+5\right) C_{r, 8,1}-\left(2 \delta^{2}-8 \sigma-10\right) C_{r, 8,0}
\end{aligned}
$$

and the subsequent terms are all obtainable from the recurrence relation. $C_{r, s, 0}$ and $C_{r, s, 1}$ can be computed directly from (17) and (35) respectively. It would be possible to develop a corresponding formula for $C_{r, s, 2}$ but it would not be very useful. Perhaps the best way to obtain $C_{r, 8,2}$ is by use of the relation (33).

It should be remarked that equation (50) is probably the most suitable, among the formulas given above, for use with an electronic computer. For work with a desk computing machine it is rather complicated, and there is little doubt that (33) would prove more useful.

4. Tables. When working by hand, it will generally be convenient to have a table of the numerical values of the $C_{\text {ret }}$. We give this immediately below in Table I for $0 \leqq r \leqq s \leqq t \leqq 10$. Some general formulas for $C_{r s t}$ as polynomials in $t$ for $0 \leqq r \leqq s \leqq 3$ are given in Table II.

5. Application. As mentioned in Section 1, our purpose was to apply the above ideas to the solution of non-linear differential equations over a semi-infinite range. 
TABLE I

\begin{tabular}{|c|c|c|c|c|c|c|c|}
\hline • & $s$ & $t$ & $C_{r s t}$ & $r$ & $s$ & $t$ & $C_{r s t}$ \\
\hline 0 & 0 & 0 & .66666667 & $\mathbf{0}$ & 5 & 8 & .07837182 \\
\hline 0 & 0 & 1 & .22222222 & 0 & 5 & 9 & .04878490 \\
\hline 0 & 0 & 2 & .07407407 & $\mathbf{0}$ & 5 & 10 & .02839004 \\
\hline $\mathbf{0}$ & 0 & 3 & .02469136 & & & & \\
\hline 0 & $\mathbf{0}$ & 4 & .00823045 & $\mathbf{0}$ & 6 & 6 & .16045055 \\
\hline 0 & 0 & 5 & .00274348 & 0 & 6 & 7 & .14305131 \\
\hline $\mathbf{0}$ & $\mathbf{0}$ & 6 & .00091449 & 0 & 6 & 8 & .11236326 \\
\hline $\mathbf{0}$ & 0 & 7 & .00030483 & 0 & 6 & 9 & .07984000 \\
\hline $\mathbf{0}$ & 0 & 8 & .00010161 & 0 & 6 & 10 & .05233831 \\
\hline 0 & 0 & 9 & .00003387 & & & & \\
\hline 0 & 0 & 10 & .00001129 & $\begin{array}{l}0 \\
0\end{array}$ & $\begin{array}{l}7 \\
7\end{array}$ & $\begin{array}{l}7 \\
8\end{array}$ & $\begin{array}{r}.14885106 \\
13475521\end{array}$ \\
\hline 0 & 1 & 1 & .37037037 & 0 & 7 & 9 & .10898616 \\
\hline 0 & 1 & 2 & .22222222 & 0 & 7 & 10 & .08038815 \\
\hline 0 & 1 & 3 & .10699588 & & & & \\
\hline $\mathbf{0}$ & 1 & 4 & .04663923 & 0 & 8 & 8 & .13945383 \\
\hline $\mathbf{0}$ & 1 & 5 & .01920439 & $\mathbf{0}$ & 8 & 9 & . 12773173 \\
\hline 0 & 1 & 6 & .00762079 & 0 & 8 & 10 & .10570201 \\
\hline 0 & 1 & 8 & .00111772 & 0 & 9 & 9 & .13163910 \\
\hline 0 & 1 & 9 & .00041773 & 0 & 9 & 10 & .12169095 \\
\hline 0 & 1 & 10 & .00015430 & & & & \\
\hline 0 & 2 & 2 & 27160494 & 0 & 10 & 10 & .12500700 \\
\hline 0 & 2 & 3 & .20027435 & 1 & 1 & 1 & -.07407407 \\
\hline 0 & 2 & 4 & .11796982 & 1 & 1 & 2 & .17283951 \\
\hline 0 & 2 & 5 & .06127115 & 1 & 1 & 3 & .21124829 \\
\hline 0 & 2 & 6 & .02936544 & 1 & 1 & 4 & .15089163 \\
\hline 0 & 2 & 7 & .01331098 & 1 & 1 & 5 & .08687700 \\
\hline 0 & 2 & 8 & .00579180 & 1 & 1 & 6 & .04440380 \\
\hline 0 & 2 & 9 & .00244242 & 1 & 1 & 7 & .02103338 \\
\hline 0 & 2 & 10 & .00100482 & 1 & 1 & 8 & .00944978 \\
\hline 0 & 3 & 3 & .22405121 & 1 & $\begin{array}{l}1 \\
1\end{array}$ & $\begin{array}{r}y \\
10\end{array}$ & $\begin{array}{l}.00408324 \\
.00171233\end{array}$ \\
\hline 0 & 3 & 4 & .18076513 & & & & \\
\hline 0 & 3 & 5 & .12000203 & 1 & 2 & 2 & -.04115226 \\
\hline 0 & 3 & 6 & .07021287 & 1 & 2 & 3 & .08504801 \\
\hline 0 & 3 & 7 & .03762977 & 1 & 2 & 4 & . 16369456 \\
\hline 0 & 3 & 8 & .01891085 & 1 & 2 & 5 & . 15333029 \\
\hline 0 & 3 & 9 & .00904835 & 1 & 2 & 6 & . 10841843 \\
\hline $\mathbf{0}$ & 3 & 10 & .00416520 & 1 & 2 & 7 & .06553879 \\
\hline 0 & 4 & 4 & .19519382 & 1 & $\begin{array}{l}2 \\
2\end{array}$ & $\begin{array}{l}0 \\
9\end{array}$ & $\begin{array}{r}.03580078 \\
01821086\end{array}$ \\
\hline 0 & 4 & 5 & .16532033 & 1 & 2 & 10 & .00878492 \\
\hline $\mathbf{0}$ & 4 & 6 & .11851174 & & & & \\
\hline $\mathbf{0}$ & 4 & 7 & .07545146 & 1 & 3 & 3 & -.02042372 \\
\hline 0 & 4 & 8 & .04399736 & 1 & 3 & 4 & .05009399 \\
\hline $\mathbf{0}$ & 4 & 9 & .02398552 & 1 & 3 & 5 & .12508256 \\
\hline 0 & 4 & 10 & .01239969 & 1 & 3 & 6 & .14086606 \\
\hline 0 & 5 & 5 & 17527816 & 1 & 3 & 8 & .11596019 \\
\hline 0 & 5 & 6 & .15303674 & 1 & 3 & 9 & .04897920 \\
\hline $\mathbf{0}$ & 5 & 7 & . 11566665 & 1 & 3 & 10 & .02762203 \\
\hline
\end{tabular}


TABLE I-Continued

\begin{tabular}{|c|c|c|c|c|c|c|c|}
\hline$r$ & s & $t$ & $C_{r s t}$ & $r$ & $s$ & $\iota$ & $C_{r s t}$ \\
\hline 1 & 4 & 4 & -.01188843 & 2 & 3 & 10 & .07467760 \\
\hline 1 & 4 & 5 & .03383631 & & & & \\
\hline 1 & 4 & 6 & .09794126 & 2 & 4 & 4 & .12284713 \\
\hline 1 & 4 & 7 & .12524062 & 2 & 4 & 5 & .04609731 \\
\hline 1 & 4 & 8 & .11552364 & 2 & 4 & 6 & -.00911108 \\
\hline 1 & 4 & 9 & .08823376 & 2 & 4 & 7 & .01571953 \\
\hline 1 & 4 & 10 & .05941345 & $\begin{array}{l}2 \\
2\end{array}$ & $\begin{array}{l}4 \\
4\end{array}$ & $\begin{array}{l}8 \\
9\end{array}$ & $\begin{array}{r}06735022 \\
09906079\end{array}$ \\
\hline 1 & 5 & 5 & -.00795949 & 2 & 4 & 10 & .10112375 \\
\hline 1 & 5 & 6 & .02489458 & & & & \\
\hline 1 & 5 & 7 & .07889869 & 2 & 5 & 5 & - . 10347339 \\
\hline 1 & 5 & 8 & .11044813 & 2 & $\stackrel{5}{5}$ & 6 & .05127568 \\
\hline 1 & 5 & 9 & .11111857 & 2 & 5 & 7 & -.00230192 \\
\hline 1 & 5 & 10 & .09212795 & $\begin{array}{l}2 \\
2\end{array}$ & $\begin{array}{l}5 \\
5\end{array}$ & $\begin{array}{l}8 \\
9\end{array}$ & $\begin{array}{l}.00330548 \\
.04431534\end{array}$ \\
\hline 1 & 6 & 6 & -.00582692 & 2 & 5 & 10 & .08081952 \\
\hline 1 & 6 & 7 & .01934154 & & & & \\
\hline 1 & 6 & 8 & .06515827 & & & & \\
\hline 1 & 6 & 9 & .09753256 & 2 & 6 & 6 & .09128849 \\
\hline 1 & 6 & 10 & . 10499757 & $\begin{array}{l}2 \\
2\end{array}$ & $\begin{array}{l}6 \\
6\end{array}$ & $\begin{array}{l}7 \\
8\end{array}$ & $\begin{array}{l}.05266394 \\
.00397312\end{array}$ \\
\hline 1 & 7 & 7 & -.00451393 & 2 & 6 & 9 & -.00198035 \\
\hline 1 & 7 & 8 & .01560049 & 2 & 6 & 10 & .02815229 \\
\hline 1 & 7 & 9 & .05492763 & & & & \\
\hline 1 & 7 & 10 & .08655013 & $\begin{array}{l}2 \\
2\end{array}$ & $\begin{array}{l}7 \\
7\end{array}$ & $\begin{array}{l}7 \\
8\end{array}$ & $\begin{array}{l}.08275543 \\
.05253757\end{array}$ \\
\hline 1 & 8 & 8 & -.00363396 & 2 & 7 & 9 & .00903873 \\
\hline 1 & 8 & 9 & .01293342 & 2 & 7 & 10 & -.00363722 \\
\hline 1 & 8 & 10 & .04709154 & ? & & 0 & 1212 \\
\hline 1 & 9 & 9 & -.00300868 & 2 & 8 & 9 & .05177168 \\
\hline 1 & 9 & 10 & .01095104 & 2 & 8 & 10 & .01298846 \\
\hline 1 & 10 & 10 & -.00254500 & 2 & 9 & 9 & .07120551 \\
\hline & & & & 2 & 9 & 10 & .05073310 \\
\hline 2 & 2 & 2 & .20576132 & 2 & 10 & 10 & .06701828 \\
\hline 2 & 2 & 3 & -.00457247 & & & & \\
\hline 2 & 2 & 4 & .00335315 & 3 & 3 & 3 & -.05375197 \\
\hline 2 & 2 & 5 & .09053498 & 3 & 3 & 4 & . 11295704 \\
\hline 2 & 2 & 6 & .13260174 & 3 & 3 & 5 & .07694175 \\
\hline 2 & 2 & 7 & .12277939 & 3 & 3 & 6 & -.00123814 \\
\hline 2 & 2 & 8 & .09060272 & 3 & 3 & 7 & .00106252 \\
\hline 2 & 2 & 9 & .05811997 & 3 & 3 & 8 & .05014082 \\
\hline 2 & 2 & 10 & .03385805 & 3 & 3 & 9 & .09001406 \\
\hline 2 & 3 & 3 & .15658182 & 0 & 0 & 10 & \\
\hline 2 & 3 & 4 & .03119443 & 3 & 4 & 4 & -.03744912 \\
\hline 2 & 3 & 5 & -.01249810 & 3 & 4 & 5 & .07177090 \\
\hline 2 & 3 & 6 & 04167161 & 3 & 4 & 6 & .08787554 \\
\hline 2 & 3 & 7 & .09805416 & 3 & 4 & 7 & .02244924 \\
\hline 2 & 3 & 8 & .11549354 & 3 & 4 & 8 & -.00731554 \\
\hline 2 & 3 & 9 & . 10130123 & 3 & 4 & 9 & .01802366 \\
\hline
\end{tabular}


TRIPLE PRODUCT INTEGRALS OF LAGUERRE FUNCTIONS

TABLE I-Continued

\begin{tabular}{|c|c|c|c|c|c|c|c|}
\hline r & $s$ & $t$ & $C_{r s t}$ & $r$ & $s$ & $t$ & $C_{r s t}$ \\
\hline 3 & 4 & 10 & .05982871 & 4 & 7 & 10 & .02567632 \\
\hline 3 & 5 & 5 & -.02226023 & 4 & 8 & 8 & .06531960 \\
\hline 3 & 5 & 6 & .04824409 & 4 & 8 & 9 & .02633548 \\
\hline 3 & 5 & 7 & .08353180 & 4 & 8 & 10 & -.00693875 \\
\hline 3 & 5 & 8 & .03995357 & & & & \\
\hline $\begin{array}{l}3 \\
2\end{array}$ & 5 & 9 & -.00156912 & 4 & $\begin{array}{l}9 \\
0\end{array}$ & 9 & .05946498 \\
\hline 3 & 5 & 10 & .00178650 & 4 & 9 & 10 & .02814148 \\
\hline 3 & 6 & 6 & -.01411745 & 4 & 10 & 10 & .05504858 \\
\hline 3 & 6 & 7 & .03516063 & & & & \\
\hline 3 & 6 & 8 & .07516477 & 5 & 5 & 5 & -.04386690 \\
\hline 3 & 6 & 9 & .04998029 & 5 & $\begin{array}{l}5 \\
5\end{array}$ & 6 & .08650875 \\
\hline 3 & 6 & 10 & .00808104 & $\begin{array}{l}5 \\
5\end{array}$ & $\begin{array}{l}5 \\
5\end{array}$ & $\begin{array}{l}7 \\
8\end{array}$ & $\begin{array}{r}.03500443 \\
-.01294078\end{array}$ \\
\hline 3 & 7 & 7 & -.00985944 & 5 & 5 & 9 & .02870872 \\
\hline 3 & 7 & 8 & .02724675 & 5 & 5 & 10 & .06403893 \\
\hline 3 & 7 & 9 & .06668946 & & & & \\
\hline 3 & 7 & 10 & .05474855 & $\begin{array}{l}5 \\
5\end{array}$ & $\begin{array}{l}6 \\
6\end{array}$ & $\begin{array}{l}6 \\
7\end{array}$ & $\begin{array}{r}-.03350414 \\
.06117194\end{array}$ \\
\hline 3 & 8 & 8 & -.00741466 & 5 & 6 & 8 & .05170139 \\
\hline 3 & 8 & 9 & .02201080 & 5 & 6 & 9 & -.00581844 \\
\hline 3 & 8 & 10 & .05915185 & 5 & 6 & 10 & .00668642 \\
\hline 3 & 9 & 9 & -.00586119 & 5 & 7 & 7 & -.02189833 \\
\hline 3 & 9 & 10 & .01830449 & $\begin{array}{l}5 \\
5\end{array}$ & $\begin{array}{l}7 \\
7\end{array}$ & $\begin{array}{l}8 \\
9\end{array}$ & .04397838 \\
\hline 3 & 10 & 10 & -.00479475 & 5 & 7 & 10 & .00564310 \\
\hline 4 & 4 & 4 & 12925988 & 5 & 8 & 8 & -.01467602 \\
\hline 4 & 4 & 5 & -.02134197 & 5 & 8 & 9 & .03341818 \\
\hline 4 & 4 & 6 & .02196251 & 5 & 8 & 10 & .05551730 \\
\hline 4 & 4 & 7 & .08246301 & & & & \\
\hline 4 & $\begin{array}{l}4 \\
4\end{array}$ & 8 & .05322789 & $\begin{array}{l}5 \\
5\end{array}$ & $\begin{array}{l}9 \\
9\end{array}$ & 9 & -.01055417 \\
\hline $\begin{array}{l}4 \\
4\end{array}$ & 4 & 10 & $\begin{array}{r}.00474524 \\
-.00303408\end{array}$ & 5 & $\boldsymbol{y}$ & 10 & .02668086 \\
\hline 4 & 5 & & 10673406 & 5 & 10 & 10 & -.00808528 \\
\hline 4 & 5 & 6 & .00196740 & 6 & 6 & 6 & 09687151 \\
\hline 4 & 5 & 7 & -.00100286 & 6 & 6 & 7 & -.02410491 \\
\hline 4 & 5 & 8 & .06117005 & 6 & 6 & 8 & .02789589 \\
\hline 4 & 5 & 9 & .06583263 & 6 & 6 & 9 & .06239762 \\
\hline 4 & 5 & 10 & .02450630 & 6 & 6 & 10 & .01401545 \\
\hline 4 & 6 & 6 & .08660706 & & & & \\
\hline 4 & 6 & 7 & .01580477 & 6 & 7 & 7 & .08320655 \\
\hline 4 & 6 & 8 & -.00811483 & 6 & 7 & 8 & -.00757894 \\
\hline 4 & 6 & 9 & .04086770 & 6 & 7 & 9 & .00739178 \\
\hline 4 & 6 & 10 & .06525394 & 6 & 7 & 10 & .05666489 \\
\hline 4 & 7 & 7 & .07367692 & 6 & 8 & 8 & .06900813 \\
\hline 4 & 7 & 8 & .02281235 & 6 & 8 & 9 & .00401100 \\
\hline 4 & 7 & 9 & -.00867086 & 6 & 8 & 10 & -.00218959 \\
\hline
\end{tabular}


TABLE I-Continued

\begin{tabular}{|c|c|c|c|c|c|c|c|}
\hline$r$ & $s$ & $t$ & $C_{r s t}$ & , & $s$ & $t$ & $C_{r s t}$ \\
\hline 6 & 9 & 9 & .05911346 & 8 & 8 & 8 & .07864342 \\
\hline 6 & 9 & 10 & .01077477 & 8 & 8 & 9 & -.02407255 \\
\hline 6 & 10 & 10 & .05253399 & & & & \\
\hline & & & & 8 & 9 & 9 & .06917670 \\
\hline $\begin{array}{l}7 \\
7\end{array}$ & $\begin{array}{l}7 \\
7\end{array}$ & $\begin{array}{l}7 \\
8\end{array}$ & $\begin{array}{r}-.03776986 \\
07123199\end{array}$ & 8 & 9 & 10 & -.01154288 \\
\hline 7 & 7 & 9 & .01652253 & 8 & 10 & 10 & .05829179 \\
\hline 7 & 7 & 10 & -.00951384 & 0 & 9 & 0 & -03353097 \\
\hline $\begin{array}{r}7 \\
7\end{array}$ & $\begin{array}{l}8 \\
8 \\
8\end{array}$ & $\begin{array}{r}8 \\
9 \\
10\end{array}$ & $\begin{array}{r}-.03035843 \\
.05351461 \\
.03227809\end{array}$ & 9 & 9 & 10 & .06114106 \\
\hline $\begin{array}{l}7 \\
7\end{array}$ & $\begin{array}{l}9 \\
9\end{array}$ & $\begin{array}{r}9 \\
10\end{array}$ & $\begin{array}{r}-.02107272 \\
.04012854\end{array}$ & $\mathbf{y}$ & 10 & 10 & -.02786041 \\
\hline 7 & 10 & 10 & -.01469083 & & & & .00001781 \\
\hline
\end{tabular}

TABLE II

\begin{tabular}{l|l|l}
\hline & $s$ & \multicolumn{1}{|c}{$C_{r s t}$} \\
\hline 0 & 0 & $2 \cdot 3^{-t-1}$ \\
& 1 & $(8 t+2) 3^{-t-2}$ \\
0 & 2 & $\left(16 t^{2}+2\right) 3^{-t-3}$ \\
0 & 3 & $\left(64 t^{3}-48 t^{2}+56 t+6\right) 3^{-t-5}$ \\
& & \\
1 & 1 & $\left(32 t^{2}-48 t+10\right) 3^{-t-3}$ \\
1 & 2 & $\left(64 t^{3}-240 t^{2}+200 t+18\right) 3^{-t-4}$ \\
1 & 3 & $\left(256 t^{4}-1664 t^{3}+3056 t^{2}-1264 t+78\right) 3^{-t-6}$ \\
2 & 2 & $\left(128 t^{4}-1024 t^{3}+2464 t^{2}-1664 t+66\right) 3^{-t-5}$ \\
2 & 3 & $\left(512 t^{5}-6528 t^{4}+28160 t^{3}-46848 t^{2}+24824 t+438\right) 3^{-t-7}$ \\
3 & 3 & $\left(2048 t^{6}-39936 t^{5}+285824 t^{4}-920832 t^{3}+1314560 t^{2}-647280 t+\right.$ \\
& & $4410) 3^{-t-9}$ \\
\hline
\end{tabular}

An example of such an application will be given to illustrate the various technical problems involved.

The Blasius Equation.

$$
\begin{gathered}
y^{\prime \prime \prime}+y y^{\prime \prime}=0 \\
y(0)=y^{\prime}(0)=0 ; \quad y^{\prime}(\infty)=2 .
\end{gathered}
$$

This is a well-known equation whose numerical solution has long been known [4]. However, as an example of the Laguerre functions method, we sought an approximate solution of the form

$$
y=f_{N}(x)=2 x+a_{N}+\sum_{r=0}^{N} b_{N r} \lambda_{r}
$$


where $a_{N}, b_{N 0}, b_{N 1}, \cdots, b_{N N}$ were constants to be determined.

The boundary condition at infinity is clearly satisfied, and, to attend to the conditions at $x=0$, we need

$$
a_{N}+\sum_{r=0}^{N} b_{N r}=0
$$

and

$$
2-\sum_{r=0}^{N}\left(r+\frac{1}{2}\right) b_{N r}=0
$$

thus leaving us the possibility of imposing $N$ further conditions on the $(N+2)$ coefficients $a_{N}, b_{N r}(r=0,1, \cdots, N)$. As an obvious set of conditions, we should substitute from (54) in (52), express $y^{\prime \prime}+y y^{\prime \prime}$ as a linear sum of $\lambda_{n}$ 's, and equate the coefficients of $\lambda_{0}, \lambda_{1}, \cdots, \lambda_{N-1}$ to zero. However, there is a difficulty in the way of this program. The expression for $\lambda_{n}{ }^{\prime}$ in terms of the Laguerre functions themselves involves all the $\lambda_{r}$ for $0 \leqq r \leqq n$, while the expressions for $\lambda_{n}{ }^{\prime \prime}, \lambda_{n}{ }^{m}$ become very complicated. The situation can be saved by pre-multiplying (52) by $x^{3}$ and making repeated use of the relation

$$
x \lambda_{n}{ }^{\prime}=\frac{1}{2}(n+1) \lambda_{n+1}-\frac{1}{2} \lambda_{n}-\frac{1}{2} n \lambda_{n-1} .
$$

Proceeding in this way, one finds oneself confronted with expressions of the form $x^{r} \lambda_{n}$. They, too, can be resolved by the repeated use of the relation

$$
x \lambda_{n}=-(n+1) \lambda_{n+1}+(2 n+1) \lambda_{n}-n \lambda_{n-1},
$$

which also follows trivially from the fundamental properties of Laguerre polynomials.

After making all of these substitutions into (52), we still have to deal with products of Laguerre functions arising from the nonlinear term, and these have to be resolved by (3). We are now in a position to equate the coefficients of $\lambda_{0}, \lambda_{1}, \cdots$, $\lambda_{N-1}$ to zero, obtaining a set of $N$ quadratic equations which, together with (55) and (56), should suffice to determine the coefficients. In general, there will be more than one solution, and any one of them might, if $N$ is large enough, be expected to yield a function $f_{N}(x)$ which will approximate the exact solution of $(52)$. Since the solutions are obtained by equating the coefficients of $\lambda_{0}, \lambda_{1}, \cdots, \lambda_{N-1}$ to zero, it has been found useful in practice to select the one for which the coefficient of $\lambda_{N}$ is least in absolute value.

Setting up the equations even for so simple a case as (52) is not a trivial task, and can become extremely laborious for more complex equations. However, there would be no real difficulty in having all the work, including the formal steps represented by (3), (57), and (58), programmed for an electronic computing machine.

We have described the procedure so far in some detail, since it is of quite general application. The next step is actually to solve the equations for $a_{N}, b_{N r}(r=0$, $1, \cdots, N)$, and for this purpose the following type of approach has been found to be practical. One first solves for some small value of $N$. The advance from $N$ to $N+1$ is effected by solving the equations for $N+1$ by the Newton-Raphson method, taking as a first approximation to this solution

$$
a_{N+1}^{(1)}=a_{N}, \quad b_{N+1, r}^{(1)}=b_{N, r} \quad(r=0,1, \cdots, N), \quad b_{N+1, N+1}^{(1)}=0 .
$$


An advantage of this choice of first approximation is that it might; for obvious reasons, be expected to be better as $N$ increases. Hence the number of steps required for convergence decreases.

In the particular case of the Blasius equation, we started with $N=1$, i.e., with three coefficients to be determined. Eliminating two of them by (55), (56) left us with a quadratic equation in one unknown. The step to $N=2$ was effected as described. There would be no difficulty in principle in carrying on to higher values of $N$. However, the arithmetic soon becomes extremely laborious, and the task is best handed over to an electronic computing machine. The result for $N=2$ is shown in Table III. The function tabulated as $f(x)$ was obtained by direct numerical integration, using essentially Hamel's original method, and is given correct to three decimal places.

\section{TABLE III}

$\begin{array}{lcc}x & f_{2}(x) & f(x) \\ 0 & 0 & 0 \\ 1 & 0.732 & 0.650 \\ 2 & 2.337 & 2.305 \\ 3 & 4.247 & 4.280 \\ 4 & 6.252 & 6.279 \\ 5 & 8.248 & 8.279 \\ 6 & 10.273 & 10.279\end{array}$

Department of Applied Mathematics

Weizmann Institute of Science

Rehovoth, Israel

1. A. Erdely1, et al., Higher Transcendental Functions, II, McGraw-Hill, New York, 1953, p. 188-189.

2. A. Erdelyi, et al., Tables of Integral Transforms, I, McGraw-Hill, New York, 1954, p. 174-175.

3. J. Gruls \& G. Weiss, "Products of Laguerre Polynomials," MTAC, v. 14, 1960, p. 60.

4. S. Goldstein, et al., Modern Developments in Fluid Dynamics, I, Clarendon Press, Oxford, 1952, p. 135.

5. G. N. Watson, Theory of Bessel Functions, University Press, Cambridge, 1948, p. 146.

6. G. N. WATsON, "A note on the polynomials of Hermite and Laguerre," J. London Math. Soc., v. 13, 1938, p. 29. 